\title{
Correlação entre os Aspectos Laparoscópicos e os Achados Histológicos das Lesões Endometrióticas Peritoneais
}

\author{
Correlation Between Laparoscopic Aspects and Histologic Findings \\ in Peritoneal Endometriotic Lesions \\ Francesco Antonio Viscomi, Rogério Dias, Laurival de Luca, \\ Mauro Fernando Kürten Ihlenfeld
}

\begin{abstract}
RESUM0
Objetivos: avaliar a correlação entre os aspectos laparoscópicos e os achados histológicos estromais incluindo, a profundidade da lesão endometriótica peritoneal, e na relação com a teoria evolutiva da endometriose.

Métodos: foram selecionadas para o estudo 67 pacientes submetidas à laparoscopia por algia pélvica, infertilidade, tumor anexial e outras indicações. A avaliação laparoscópica baseou-se no aspecto visual do implante suspeito de endometriose peritoneal, o qual foi biopsiado. De acordo com o aspecto laparoscópico, as lesões foram agrupadas em: grupo $V$ lesões vermelhas, grupo $N$ - lesões negras e grupo $B$ - lesões brancas. Os parâmetros histológicos estudados foram: profundidade da lesão, presença de hemossiderina no estroma, vascularização estromal e presença de fibrose no estroma.

Resultados: a profundidade da lesão mostrou diferenças estatisticamente significantes entre os grupos de estudo. As lesões vermelhas mostraram-se superficiais em 100\% dos casos. As lesões negras apresentaram-se superficiais em 55,6\%, intermediárias em 38,9\% e profundas em 5,5\%. As lesões brancas mostraram-se superficiais em $28 \%$, intermediárias em $68 \%$ e profundas em 4\%. A presença de hemossiderina no estroma se mostrou equivalente nos 3 grupos. A presença de vasos no estroma da lesão endometriótica, que foi classificada de I a III de acordo com a quantidade, demonstrou diferenças significantes entre os 3 grupos, sendo que a vascularização exuberante (grau III) esteve presente em $60 \%$ das lesões vermelhas $e$ em 10\% das lesões brancas. A presença de tecido fibrótico na lesão endometriótica apresentou diferenças estatisticamente significantes nos 3 grupos de estudo, sendo mais freqüente no grupo B (lesões brancas), com 70,6\%.

Conclusão: as variáveis analisadas nos diferentes grupos de estudo demostraram diferença significantes entre os grupos, reforçando a teoria evolutiva da endometriose peritoneal.
\end{abstract}

PALAVRAS-CHAVE: Endometriose. Laparoscopia. Hemossiderina. Endométrio. Dor pélvica.

Introdução

A endometriose é definida como condição na qual um tecido com estrutura histológica e resposta fisiológica semelhantes às do endométrio ocorre em localização ectópica, principalmente na pelve $^{1}$, e é considerada uma das doenças gineco-

Departamento de Ginecologia e Obstetricia, Faculdade de Medicina de Botucatu, Universidade Estadual Paulista UNESP

Correspondência: Francesco Antonio Viscomi

Avenida Eusébio Matoso, 196

05423-000 - São Paulo - SP

e-mail: fviscomi@womens.com.br lógicas benignas mais comuns na idade reprodutiva da mulher. Aproximadamente 10 a $15 \%$ das pacientes apresentam sintomas, especialmente dor, que demandam a instituição de um tratamento. Wheeler ${ }^{1}$ demonstrou que a mesma pode estar presente ao redor de $10 \%$ na idade reprodutiva, a partir de achados cirúrgicos em mulheres que não apresentavam sintomas ${ }^{2}$.

Recentes relatos questionam se todo tecido endometrial ectópico deve ser considerado patológico, reservando esta definição para os casos nos quais ocorra progressão e dano tecidual associados a seus respectivos sinais e sintomas ${ }^{3}$. Os sin- 
tomas de pacientes com endometriose são extremamente variados. Estes podem variar dependendo do local onde se implantou a lesão e nem sempre guarda uma relação direta com a extensão da doença. Em outras ocasiões, a endometriose é um achado cirúrgico de investigação de outras doenças. Não há relação entre o aspecto da lesão e a sintomatologia ${ }^{4}$, mas em relação à profundidade, a endometriose profunda está fortemente associada à dor pélvica ${ }^{5}$.

O diagnóstico laparoscópico da endometriose peritoneal habitualmente é feito pelo reconhecimento das lesões típicas, caracterizadas pela coloração escura tipo "chamuscado de pólvora". Essas lesões negras são resultado de sangramentos cíclicos e retenção do pigmento sangüíneo refletindo a cor da lesão, responsável por sua aparência tipica.

Jansen e Russell ${ }^{6}$ descrevem outras características visuais de lesões peritoneais, como: opacificações peritoneais, lesões vermelhas em "chama de vela", excrescências glandulares, aderências subovarianas e lesões amarelopardacentas, denominando-as como "não pigmentadas" ou simplesmente atípicas. Outros aspectos importantes incluem lesões polipóides avermelhadas, lesões de hipervascularização, lesões petequiais, opacificações esbranquiçadas, aderências e defeitos peritoneais. As lesões atipicas tendem a ser mais freqüentes, conforme relatos de Redwine $^{7}$ que encontrou lesões típicas em $60 \%$ e atípicas em $66 \%$ de 137 pacientes portadoras de endometriose com múltiplas lesões.

As lesões da endometriose peritoneal de acordo com seu aspecto macroscópico, são classificadas em negras, vermelhas e brancas. Os implantes endometrióticos variam de ativos a inativos e estes estágios podem ser reconhecidos visualmente $^{4}$. Nisolle et al. ${ }^{8}$ concluiram que as lesões vermelhas são ativas e podem representar o estádio inicial da implantação da lesão. As lesões vermelhas costumam se localizar mais freqüentemente nos ligamentos largos e nos útero-sacros. A aparência laparoscópica costuma ser de uma lesão superficial com coloração e translucência semelhantes à mucosa endometrial vista durante um exame histeroscópico. As lesões vermelhas também podem ser representadas por áreas peritoneais de hipervascularização e petéquias ${ }^{8}$. Estas lesões costumam localizar-se na reflexão vésico-uterina e no ligamento largo.

Em contraste com as anteriores, as lesões negras demonstram inatividade e formas regressivas. A progressiva descamação menstrual provocaria uma reação inflamatória com escarificação local. A presença de debris menstruais circunscritos numa área de peritôneo é responsável pela aparência negra típica ${ }^{8}$.

As lesões brancas têm aparência de opacificações peritoneais e habitualmente são lesões mais espessas. Outro aspecto é representado pelas aderências, que, ao contrário das seqüelas de salpingite ou peritonite, têm localização entre o ovário e o peritôneo na fosseta ovárica ${ }^{8}$.

Diferentes tipos de crescimento e evolução são descritos, de acordo com a aparência macroscópica da lesão. Redwine ${ }^{9}$ sugere uma correlação da atividade do implante peritoneal com a idade da paciente. Esse autor quantificou em 7 a 10 anos o prazo para que uma lesão mude da aparência avermelhada para o aspecto negro cicatricial. Esta alteração foi também observada por Koninckx et al. ${ }^{5}$ que documentaram um aumento de $23 \%$ para $63 \%$ na ocorrência de lesões cicatriciais após os 20 anos de idade e demonstrou uma diminuição na ocorrência de lesões vermelhas polipóides e um aumento na ocorrência de lesões profundas. A relação do tipo de implantes com a atividade da doença foi relatada por Donnez et al. ${ }^{10}$.

Somente com a presença de glândulas endometriais e estroma na avaliação histológica o diagnóstico de endometriose é confirmado. As técnicas histológicas podem demonstrar se a implantação endometriótica é ativa e infiltra os tecidos vizinhos ou se é superficial e inativa.

Histologicamente as lesões vermelhas mostram a presença de numerosas glândulas endometriais ativas e estroma bem vascularizado semelhante ao endométrio tópico. As lesões negras, as quais são resultado de sangramento tecidual e retenção de pigmento sangüíneo, macroscopicamente apresentam-se como áreas escurecidas e histologicamente apresentam glândulas, estroma e debris intraluminais com fenômenos degenerativos. As lesões brancas caracterizam-se visualmente por opacificações, aderências subovarianas, lesões amarelo-pardacentas e defeitos peritoneais. Histologicamente compõem-se de estruturas glandulares retroperitoneais circundadas por estroma escasso com tecido fibroso ou conjuntivo.

Apesar da importância dada nas últimas décadas ao aspecto macroscópico das lesões, a profundidade de infiltração da lesão endometriótica também desempenha um papel importante na sintomatologia da paciente ${ }^{4}$. Em relação à profundidade, a lesão endometriótica pode ser superficial, quando a infiltração ocorre até $2 \mathrm{~mm}$ abaixo da superficie do peritônio, intermediária, de 2 a $5 \mathrm{~mm}$, e lesão endometriótica profunda, definida como sendo aquela que infiltra mais de $5 \mathrm{~mm}$ na profundidade $^{11}$. As lesões profundas são aquelas que estariam mais associadas à dispareunia e à algia pélvica $^{5}$. As lesões endometrióticas intermediári- 
as e profundas provocam a persistência dos sintomas dolorosos até que a exérese total da lesão seja realizada. Os implantes são excisados utilizandose eletrocautério monopolar, tesoura, bisturi ultra-sônico ou laser de $\mathrm{CO}_{2}$.

Qualquer que seja o instrumental a ser utilizado, a técnica permanece a mesma. É realizada uma incisão circular, apreende-se o peritônio normal em volta do implante com uma pinça preensora e procede-se então à dissecção do peritônio e da gordura retroperitoneal, observando se a lesão está sendo retirada na sua profundidade.

O objetivo do presente estudo é avaliar a correlação entre os aspectos laparoscópicos com os achados histológicos estromais e a profundidade da lesão endometriótica peritoneal, considerando a teoria evolutiva da endometriose. A partir desta teoria, torna-se possivel o melhor entendimento da evolução das lesões, a fim de que seja possivel o reconhecimento dos tipos de lesões e a instituição do tratamento mais adequado para cada caso.

\section{Pacientes e Métodos}

Trata-se de estudo prospectivo, transversal e analítico. Foram selecionadas 67 pacientes submetidas à videolaparoscopia. As indicações da videolaparoscopia foram algia pélvica (41 casos), infertilidade (17 casos), tumor anexial (5 casos) e outras doenças, que somaram 4 casos. A faixa etária variou de 15 a 45 anos, sendo a média igual a 32,3 anos, com desvio padrão de 6,5 anos. A média de paridade foi de 0,4 , variando de zero ao máximo de 2 . As correlações entre as variáveis idade e paridade com os grupos estudados não demonstraram diferença estatisticamente significante (Tabela 1).

Tabela 1 - Proporção das variáveis idade e paridade nos grupos de estudo.

\begin{tabular}{lccccc}
\hline Grupos & & Idade & & \multicolumn{2}{c}{ No. partos } \\
& Média & DP & Variação & $\mathbf{0}$ & $\mathbf{1}$ \\
\hline V & 31,8 & 8,91 & $15-45$ & 19 & 3 \\
N & 31,4 & 4,34 & $23-42$ & 15 & 3 \\
B & 33,9 & 4,90 & $23-42$ & 14 & 5 \\
\hline
\end{tabular}

Grupos V - lesões vermelhas; $N$ - lesões negras; B - lesões brancas

Teste exato de Fisher (2-tail) $p>0,05$ (n.s.)

Os critérios de seleção das pacientes foram presença de endometriose peritoneal, disponibilidade de obter as lâminas para confirmação diagnóstica e avaliação uniforme dos aspectos histológicos. As pacientes com endometriose peritoneal foram divididas em 3 grupos, segundo o aspecto laparoscópico da lesão peritoneal: Grupo $\mathrm{V}$ - lesão vermelha: pacientes que apresentavam lesões que compreendiam pólipos avermelhados, hipervascularização, petéquias e excrescências glandulares; Grupo N - lesão negra: pacientes que apresentavam lesões escuras, rodeadas ou não de áreas cicatriciais; Grupo B - lesão branca: pacientes que apresentavam lesões de opacificação peritoneal, lesões "café-au-lait", aderências na fosseta ovárica e falhas peritoneais.

O diagnóstico de endometriose peritoneal foi realizado por meio da laparoscopia e confirmado pela avaliação histopatológica. Pacientes nas quais se encontrou mais de um aspecto de lesão endometriótica não foram consideradas no presente estudo.

Todas as pacientes foram submetidas à anestesia geral, entubadas e com respiração con- trolada. Foram realizadas 3 incisões: a primeira, de $10 \mathrm{~mm}$, na cicatriz umbilical, e outras duas, de $5 \mathrm{~mm}$, nas fossas iliacas direita e esquerda. O pneumoperitônio foi realizado com a introdução de $\mathrm{CO}_{2}$ na cavidade abdominal até atingir pressão de insuflação em torno de 12 a $14 \mathrm{mmHg}$. Introduzia-se o conjunto de óptica e endocâmera (Karl Storz, Tuttlingen, Alemanha) através da incisão da cicatriz umbilical. Após a introdução das pinças auxiliares, um rigoroso inventário do peritônio abdominal e pélvico foi realizado, com o objetivo de verificar a presença de lesões suspeitas de endometriose para posterior realização de biópsia.

As biópsias foram obtidas de áreas de peritônio pélvico suspeito de endometriose. A apreensão da lesão suspeita foi feita com pinça preensora de $5 \mathrm{~mm}$ (Karl Storz, Tuttlingen, Alemanha), seguida de uma incisão circular em torno da lesão com uma tesoura laparoscópica e retirada da lesão.

Os espécimes de biópsias obtidos foram identificados com relação ao local e aspecto. Foram fixados em formaldeído e incluidos em parafina. Os cortes histológicos foram corados com 
hematoxilina-eosina. O prerequisito para o diagnóstico histológico de endometriose foi o achado de glândula endometrial ou estroma, ou ambos. Todos os casos foram revisados em conjunto com um único médico patologista.

Os exames histológicos do estroma foram realizados, sendo observados parâmetros de presença de hemossiderina e debris, tecido fibromuscular e vascularização. A presença de hemossiderina no estroma foi quantificada em 4 graus: grau 0 (ausência de hemossiderina); grau I (presença focal de pequenos agregados de macrófagos com hemossiderina); grau II (situação intermediária) e grau III (presença multifocal de numerosos macrófagos carregados com hemossiderina).

Para padronizar esta avaliação, utilizou-se a reação de coloração com o azul da Prússia em dois casos de cada situação.

O número de vasos no estroma foi quantificado verificando 10 campos ópticos de microscópio e dividido em 3 graus: grau I vascularização pouco evidente, caracterizada pela presença eventual de pequenos vasos, pouco túrgidos, no seio e na periferia da lesão; grau II - situação intermediária e grau III - vascularização exuberante, caracterizada por numerosos vasos de pequeno calibre, espalhados por toda a lesão, em geral túrgidos, com freqüentes focos de hemorragia recente.

Na avaliação da profundidade da lesão foram utilizados os parâmetros de distribuição da profundidade, de acordo com Martin et al. ${ }^{4}$. Esses autores denominam de superficiais lesões de até $2 \mathrm{~mm}$ de profundidade, de intermediárias as lesões entre 2 e $5 \mathrm{~mm}$ e como profundas as lesões que ultrapassam $5 \mathrm{~mm}$.

Medidas de profundidade da lesão foram realizadas por meio de sistema computadorizado digital de imagem Kontron Imaging System (Carl Zeiss, Jena, Alemanha). Foram estabelecidos dois parâmetros. o primeiro, representado pela superfície do mesotélio, e o segundo, pela última glândula visualizada. De acordo com o vetor de medida do programa, foram obtidos os resultados em micrômetros.

A análise estatística foi realizada inicialmente com todas as variáveis analisadas descritivamente. Para as variáveis contínuas, como idade e paridade, esta análise foi feita por meio da observação dos valores mínimos e máximos e do cálculo de médias e desvios padrão. Para as variáveis classificatórias, calcularam-se as freqüências absolutas e relativas. A comparação entre proporções foi avaliada através do teste $\chi^{2}$ e do teste exato de Fisher. O nivel de significância utilizado para os testes foi de $5 \%$.
O projeto de pesquisa do presente estudo foi aprovado pela Comissão de Ética em Pesquisa da Faculdade de Medicina de Botucatu da Universidade Estadual Paulista - UNESP.

\section{Resultados}

A profundidade da lesão foi classificada em superficial, intermediária e profunda. As lesões vermelhas mostraram-se superficiais em 100\% dos casos. As lesões negras apresentaram-se superficiais em 55,6\%, intermediárias em 38,9\% e profundas em 5,5\%. As lesões brancas mostraramse superficiais em 28\%, intermediárias em 68\% e profundas em 4\% (Tabela 2).

Tabela 2 - Profundidade das lesões nos grupos de estudo.

\begin{tabular}{lcccc}
\hline Grupo & Superficial & Intermediária & Profunda & Total \\
\hline $\mathrm{V}[\mathrm{n}(\%)]$ & $24(100)$ & $0-$ & 0 & 24 \\
$\mathrm{~N}[\mathrm{n}(\%)]$ & $10(55,5)$ & $7(38,8)$ & $1(5,5)$ & 18 \\
$\mathrm{~B}[\mathrm{n}(\%)]$ & $7(28,0)$ & $17(68,0)$ & $1(4,0)$ & 25 \\
\hline
\end{tabular}

Grupos V - lesões vermelhas; $\mathrm{N}$ - lesões negras; B - lesões brancas Teste exato de Fisher (2-tail) $p=0,001$

Houve comportamento semelhante quanto à presença de hemossiderina no estroma nos 3 diferentes grupos, não havendo diferença estatisticamente significante (Tabela 3).

Tabela 3 - Graus de intensidade da presença de hemossiderina no estroma.

\begin{tabular}{lccllc}
\hline Grupo & Grau 0 & Grau I & Grau II & Grau III & Total \\
\hline V [n(\%)] & $12(50)$ & $5(20,8)$ & $4(16,7)$ & $3(12,5)$ & 24 \\
N [n(\%)] & $6(33,3)$ & $3(16,7)$ & $4(22,2)$ & $5(27,8)$ & 18 \\
B [n(\%)] & $18(72,0)$ & $5(20,0)$ & $1(4,0)$ & $1(4,0)$ & 25 \\
\hline
\end{tabular}

Grupos $V$ - lesões vermelhas; $N$ - lesões negras; B - lesões brancas Grau 0 - ausência de hemossiderina; I - presença focal; II - intermediária; III - presença multifocal

Teste exato de Fisher (2-tail) $p>0,05$

Quando se comparou a presença de vasos no estroma nos Grupos V, N e B, houve diferença estatisticamente significante entre eles. Em 60\% das pacientes que apresentaram o maior número de capilares (grau III), as lesões eram pertencentes ao Grupo V. Quando analisadas todas as lesões do Grupo V, 60\% delas apresentaram a maior quantidade de vasos (Tabela 4).

A presença de tecido fibrótico substituindo o estroma foi observada e mostrou variação entre 
os grupos estudados. A presença de maior quantidade de tecido fibrótico foi encontrada no Grupo B. Houve diferença estatisticamente significante quando se comparou a presença de tecido fibrótico nos diferentes grupos de lesão (Tabela 5).

Tabela 4 - Graus de vascularização do estroma das lesões dos grupos de estudo.

\begin{tabular}{lrrcc}
\hline Grupo & Grau I & Grau II & Grau III & Total \\
\hline V [n(\%)] & $2(10,0)$ & $6(30,0)$ & $12(60,0)$ & 20 \\
N [n(\%)] & $8(42,1)$ & $5(26,3)$ & $6(31,6)$ & 19 \\
B [n(\%)] & $16(57,1)$ & $10(35,7)$ & $2(7,2)$ & 28 \\
\hline
\end{tabular}

Grupos V - lesões vermelhas; $\mathrm{N}$ - lesões negras; B - lesões brancas

Graus I - vascularização pouco evidente; II - intermediária; III - vascularização exuberante

Teste $\chi^{2}(15,647$, grau de liberdade 4) $p=0,004$

Tabela 5 - Presença de tecido fibrótico no estroma das lesões dos grupos de estudo.

\begin{tabular}{lccc}
\hline Grupo & Ausente & Presente & Total \\
\hline$V[n(\%)]$ & $22(91,7)$ & $2(8,3)$ & 24 \\
N $[n(\%)]$ & $10(55,6)$ & $8(44,4)$ & 18 \\
$B[n(\%)]$ & $1(4,0)$ & $24(96,0)$ & 25 \\
\hline
\end{tabular}

Grupos V - lesões vermelhas; $\mathrm{N}$ - lesões negras; $\mathrm{B}$ - lesões brancas

Teste $\chi^{2}(38,042$, grau de liberdade 2) $p=0,001$

\section{Discussão}

Embora a endometriose seja uma doença primariamente da mulher em idade reprodutiva, são descritos casos em mulheres na adolescência e na pós-menopausa. Em nosso estudo, a média de idade das pacientes foi de 32,3 anos variando de um minimo de 15 a um máximo de 45 anos. $\mathrm{Na}$ análise comparativa da idade entre os grupos de estudo $\mathrm{V}, \mathrm{N}$ e $\mathrm{B}$, não houve diferença significante, mostrando que não há associação entre o aspecto das lesões endometrióticas peritoneais e a idade, concordando com a literatura ${ }^{12}$.

Em relação à paridade, investigando os fatores que predispõem a mulher para a endometriose, encontraram que a nuliparidade prolongada foi um fator de risco significante. Nossos resultados revelaram que quanto à paridade das pacientes, não houve diferença significante entre os três grupos estudados.

Quanto à profundidade da lesão, a endometriose profunda é definida como sendo a lesão que infiltra mais que $5 \mathrm{~mm}$ a partir do mesotélio peritoneal ${ }^{5}$. Poucos trabalhos na literatura abordam a correlação entre o aspecto da lesão endometriótica peritoneal e a profundidade.
Nisolle e Donnez ${ }^{13}$ relataram que as lesões vermelhas se localizam freqüentemente na superfície do peritôneo. No entanto, a correlação dos tipos de lesão (vermelhas, negras e brancas) com a profundidade não foi confirmada por estes autores. Koninckx e Martin ${ }^{14}$ relatam que a profundidade da lesão não pode ser antecipada pelo aspecto da mesma.

Em nossos resultados, a correlação entre a profundidade e os diferentes aspectos das lesões endometriais mostrou-se significante. Todas as lesões vermelhas foram superficiais, ao passo que $55,6 \%$ das lesões negras e $28 \%$ das brancas eram superficiais, evidenciando uma gradual invasão na profundidade de acordo com o aspecto da lesão endometriótica.

Quanto à presença de hemossiderina, segundo Nisolle et al. ${ }^{8}$, as lesões negras resultam de um sangramento tecidual e retenção do pigmento vermelho, produzindo o aspecto de coloração típica da lesão. A presença de hemorragia local no estroma poderia representar o início da fibrose no implante endometriótico ${ }^{15}$. Em nossos casos, as diferenças quanto à presença de hemossiderina no estroma não se mostraram estatisticamente significantes nos três grupos de estudo.

No que diz respeito à vascularização estromal do implante de endometriose, Nisolle et al. ${ }^{8}$ demonstraram que há uma significante diferença entre as lesões vermelhas, negras e brancas, sendo que as primeiras apresentam vascularização mais abundante e as últimas, a menor presença de vasos no estroma. A vascularização estromal se mostrou similar nas lesões vermelhas e no endométrio tópico. Isso sugere uma angiogênese induzida por implantação recente pela influência de fatores de crescimento e citocinas ${ }^{16}$.

Ferriani et al. ${ }^{17}$ detectaram fatores de crescimento de fibroblastos no tecido endometrial tópico e ectópico, sugerindo seu papel na proliferação e angiogênese do endométrio tópico e ectópico. Nossos resultados mostraram uma diferença significante quando se comparou a presença de vasos no estroma dos grupos $\mathrm{V}, \mathrm{N}$ e B. Os casos classificados como grau III correspondem a 60\% das lesões vermelhas, 30\% das lesões negras e 10\% das brancas, demonstrando concordância com os dados da literatura. Houve um decréscimo gradual na vascularização do estroma respectivamente nas lesões vermelhas, negras e brancas.

A origem da fibrose não está esclarecida, mas pode estar associada com a alta prevalência de hemorragia local no implante endometriótico ${ }^{15}$. O processo inflamatório é provavelmente o responsável pela redução da vascularização, demonstrado pela significativa redução das áreas de superfi- 
cie relativas aos capilares e ao estroma ${ }^{8}$. Histologicamente a lesão endometriótica que está circundada por tecido fibroso pode variar de graus e tem seu estroma substituido pela fibrose ${ }^{18}$. Nossos resultados mostraram que houve associação entre o tipo de lesão e a presença de tecido fibroso. Nos casos em que se encontrou a presença de fibrose, a distribuição nos grupos $\mathrm{V}, \mathrm{N}$ e B foi respectivamente de $5,7 \%, 23,5 \%$ e $70,5 \%$. A presença de tecido fibrótico foi significativamente maior nas lesões brancas quando comparada com as lesões vermelhas e negras, mostrando a caracteristica reparativa deste tipo de lesão, concordando com a literatura.

Em resumo, observamos que as lesões vermelhas mostraram-se superficiais, ricamente vascularizadas e com estroma frouxo, característico do endométrio tópico. As lesões negras apresentaram-se por vezes superficiais e por vezes intermediárias, moderadamente vascularizadas e com aparecimento de fibrose no estroma. As lesões brancas mostraram-se na maioria das vezes intermediárias em relação à profundidade, com escassa vascularização e estroma escasso, apresentando intensa fibrose.

Este estudo vem reforçar a teoria evolutiva dos implantes de endometriose peritoneal, cujo processo teria início pelas lesões vermelhas, que posteriormente evoluiriam para lesões negras, para finalmente adquirirem o aspecto de lesões brancas. Durante a evolução na aparência do implante endometriótico peritoneal, ocorreria simultaneamente um processo de invasão do peritônio em profundidade.

\section{ABSTRACT}

Purpose: to evaluate the correlation between the laparoscopic aspects and the stromal histologic findings of peritoneal endometriosis in order to understand the evolutive theory of endometriosis.

Methods: sixty-seven women were submitted to laparoscopy for pelvic pain, infertility, ovarian tumor and other pathologies. A peritoneal biopsy was taken from the typical (puckered black) and atypical endometriotic implants. The different aspects of endometriosis were classified as follows: red lesions (Group V), black lesions (Group N) and white lesions (Group B). The histological sections were examined according to a standardized protocol. The histologic parameters used were: depth of the lesion, presence of hemosiderin, vascularization of the stroma and fibrotic tissue in stroma.

Results: regarding lesion depth, there were significant differences between the groups. Red lesions were located consistently on the surface of the peritoneum (100\%) and black lesions were superficial in 55.6\%, intermediate in $38.9 \%$ and deep in 5.5\%. White lesions were superficial in $28 \%$, intermediate in $68 \%$ and deep in $4 \%$. The presence of hemosiderin showed equivalent results in the 3 groups. The large stromal vascularization was present in the red lesions (60\%), which a statistically significant difference compared to the other groups. Fibrotic tissue was present in $70.6 \%$ of the white lesions (group B), a fact that was significantly different when compared to groups $V$ and $N$.

Conclusion: the parameters analyzed in this study confirmed the importance of the evolutive theory of endometriosis.

KEY WORDS: Endometriosis. Laparoscopy. Pelvic pain. Hemosiderin.

\section{Referências Bibliográficas}

1. Wheeler JM. Epidemiology of endometriosis-associated infertility. J Reprod Med 1989; 34:41-6.

2. Houston DE. Evidence for the risk of pelvic endometriosis by age, race and socioeconomic status. Epidemiol Rev 1984; 6:167-91.

3. Stripling MC, Martin DC, Chatman DL, Van der Zwaag R, Poston WM. Subtle appearance of pelvic endometriosis. Fertil Steril 1988; 49:427-32.

4. Martin DC, Hubert GD, Van der Zwaag R, El-Zeky FA. Laparoscopic appearances of peritoneal endometriosis. Fertil Steril 1989; 51:63-7.

5. Koninckx PR, Meuleman C, Demeyere S, Lesaffre E, Cornillie FJ. Suggestive evidence that pelvic endometriosis is a progressive disease, whereas deeply infiltrating endometriosis is associated with pelvic pain. Fertil Steril 1991; 55:759-65.

6. Jansen RP, Russell P. Nonpigmented endometriosis: clinical, laparoscopic, and pathologic definition. Am J Obstet Gynecol 1986; 155:1154-9.

7. Redwine DB. The distribution of endometriosis in the pelvis by age groups and fertility. Fertil Steril 1987; 47:173-5.

8. Nisolle M, Casanas-Roux F, Anaf V, Mine JM, Donnez J. Morphometric study of the stromal vascularization in peritoneal endometriosis. Fertil Steril 1993; 59:681-4.

9. Redwine DB. Age-related evolution in color appearance of endometriosis. Fertil Steril 1987; 48:1062-3.

10.Donnez J, Nisolle M, Casanas-Roux F. Threedimensional architectures of peritoneal endometriosis. Fertil Steril 1992; 57:980-3.

11.Cornillie FJ, Lauweryns JM, Seppala M, Riittinen L, Koninckx PR. Expression of endometrial protein PP14 in pelvic and ovarian endometriotic implants. Hum Reprod 1991; 6:1411-5. 
12.Goldstein DP, De Cholnoky C, Emans SJ, Leventhal JM. Laparoscopy in the diagnosis and management of pelvic pain in adolescents. J Reprod Med 1980; 24:251-6.

13.Nisolle M, Donnez J. Peritoneal endometriosis, ovarian endometriosis and adenomyotic nodules of the rectovaginal septum are three different entities. Fertil Steril 1997; 68:585-96.

14.Koninckx PR, Martin DC. Deep endometriosis: a consequence of infiltration or retraction or possibly adenomyosis externa? Fertil Steril 1992; 58:924-8.

15. Metzger DA, Olive DL, Haney AF. Limited hormonal responsiveness of ectopic endometrium: histologic correlation with intrauterine endometrium. Human Pathol 1988; 19:1417-24.
16.Smith SK. Vascular endothelial growth factor and the endometrium. Hum Reprod 1996; 11 Suppl. 2:56-61.

17.Ferriani RA, Charnok-Jones DS, Prentice A, Thomas EJ, Smith SK. Immunohistochemical localization of acidic and basic fibroblast growth factors in normal human endometrium and endometriosis and the detection of their mRNA by polymerase chain reaction. Hum Reprod 1993; 8:11-6.

18.Bergqvist A, Ljungberg O, Myhre E. Human endometrium and endometriotic tissue obtained simultaneously: a comparative histological study. Int J Gynecol Pathol 1984; 3:135-45.

\section{Jornada Pernambucana de Ginecologia e Obstetrícia}

\section{3 a 25 de maio de 2002}

RECIFE-PE

\section{Informações:}

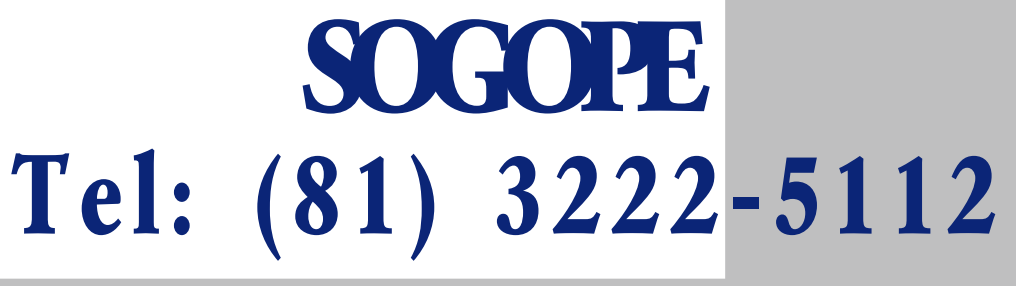

